\title{
PSEUDO-RIEMANNIAN METRIC SINGULARITIES AND THE EXTENDABILITY OF PARALLEL TRANSPORT
}

\author{
MAREK KOSSOWSKI ${ }^{1}$
}

\begin{abstract}
We are given a $C^{\infty}$ immersion $i: N \rightarrow(M,\langle\rangle)$, and $p \in N$ is a point where $N_{p}{ }^{\perp} \cap T_{p} N$ is one-dimensional. We have shown that there is a tensor $\mathrm{II}_{p}$ : $T_{p} N \times T_{p} N \times \operatorname{Rad}_{p} \rightarrow \mathbf{R}$ intrinsic to $\left(N, i^{*}\langle\rangle\right)$ which determines an extrinsic feature of the immersion. The purpose of this paper is to show that II controls the following two intrinsic properties. First, II determines which pairs of vector fields $X$, $Y$ on $N$ have the property that intrinsic covariant derivative $\nabla_{X} Y$ extends smoothly to all of $N$. Second, given a curve in $N$ containing $p, \mathrm{II}_{p}$ determines which parallel vector fields along the curve extend smoothly through $p$. As an application we locally characterize product and flat metric singularities.
\end{abstract}

This paper is a sequel to [4] and assumes the reader is familiar with Propositions 1-5 which appear there. We were given a $C^{\infty}$ immersions $i: N \rightarrow(M,\langle\rangle)$ of smooth manifolds with dimension $n$ and $m$ respectively. $M$ carries a smooth nondegenerate pseudo-Riemannian metric and $p \in N$ is a point where $N^{\perp} \cap T_{p} N$ is one-dimensional. That is, $i *\langle\rangle_{p}$ has rank $n-1$. (Recall that any compact hypersurface of a Lorentzian vector space must have such a point.) In [4] we showed that there is a tensor $\mathrm{II}_{p}: T_{p} N \times T_{p} N \times \operatorname{Rad}_{p} \rightarrow \mathbf{R}$ intrinsic to $(N, i *\langle>)$ which determines an extrinsic feature of the immersion. The purpose of this paper is to show that II controls the following two intrinsic properties. First (Theorem 6) II determines which pairs of vector fields $X, Y$ on $N$ have the property that intrinsic covariant derivative $\nabla_{X} Y$ extends smoothly to all of $N$. Second (Theorem 7) given a curve in $N$ containing $p, \mathrm{II}_{p}$ determines which parallel vector fields along the curve extend smoothly through $p$. Theorem 6 follows directly from geometric considerations. The proof of Theorem 7 reduces to the qualitative theory of normally hyperbolic vector fields (see the appendix and [2]). As an application of Theorem 7 we locally characterize product and flat metric singularities (Theorems 8 and 9). We close with three simple examples.

The author would like to thank J. Damon, P. Eberlein, R. B. Gardner, M. Schlessinger, and J. Stasheff for inspiration and Rice University for its support.

All terminology and notation will be carried over from [4]. Since our results concern only the induced metric on the submanifold we will write $(N,\langle\rangle)$ for $(N, i *\langle\rangle)$. We will assume that the metric singularities of $(N,\langle\rangle)$ are all transverse

Received by the editors April 8, 1985 and, in revised form, October 23, 1985.

1980 Mathematics Subject Classification (1985 Revision). Primary 53B30, 53B25; Secondary 83C99.

${ }^{1}$ Research partially supported by NSF Grant DMS 83-52009. 
of codimension one. So the singular locus $S \subset N$ is a codimension one submanifold. At a point $p$ in $S$ we have the radical subspace, $\operatorname{Rad}_{p}$ of $T_{p} N$. It is the one-dimensional subspace which is \langle\rangle$_{p}$-orthogonal to all of $T_{p} N$. $\left(R_{p} \in \operatorname{Rad}_{p}\right.$ will denote an arbitrary element of this subspace.) The dual connection of $(N,\langle\rangle)$ and the Levi-Civita connection of $(N-S,\langle\rangle)$ will be denoted by $\square$ and $\nabla$ respectively. Recall that the dual connection of $(N,\langle\rangle)$ is a weakened version of the classical Levi-Civita connection. It is defined at all points of $N$. It differs from the classical Levi-Civita connection in that it takes values in the 1-forms on $N$ rather than vector fields. If the radical is everywhere transverse to $S$ then the induced metric on $S$ is nondegenerate. In this case ${ }^{S} \nabla$ will denote its Levi-Citiva connection.

THEOREM 6. Given $(N,\langle\rangle)$ with all metric singularities transverse of codimension one, let $X, Y \in V^{\infty}(N)$ be two vector fields on $N$ then $\nabla_{X} Y$ extends smoothly to all of $N$ if and only if $\operatorname{II}_{p}(X, Y, R)=0$ for all $p$ in the singular locus $S$.

Proof. We will need the following lemma which is a simple adaptation of the Gram-Schmidt algorithm. Its proof is omitted.

Lemma 6.1. Given $(N,\langle\rangle)$ with \langle\rangle$_{p}$ of type $(r, s), r+s=n-1$, and $e_{1}, \ldots, e_{n}$ a local $C^{\infty}$ frame at $p$ with $e_{n}(p) \subset \operatorname{Rad}_{p}$, then there exists a local $C^{\infty}$ frame $f_{1}, \ldots, f_{n}$ such that $\operatorname{span}\left(e_{1}, \ldots, e_{i}\right)=\operatorname{span}\left(f_{1}, \ldots, f_{i}\right)$ for $1 \leqslant i \leqslant n$ and \langle\rangle is represented by

$$
\sum_{i=1}^{m-1} \pm f_{i}^{*} \odot f_{i}^{*}+g \cdot f_{m}^{*} \odot f_{m}^{*},
$$

with $g$ smooth and $g(p)=0 .(\odot$ denotes symmetric tensor product. $)$

We will refer to such a frame as a radical adapted orthonormal frame.

Proof of Proposition 6. Let $e_{1}, \ldots, e_{n}$ be a local orthonormal radical adapted frame at $p$. It is clear that all $\left\langle\nabla_{X} Y, e_{i}\right\rangle=\square_{X} Y\left(e_{i}\right)$ are smooth. Now,

$$
\nabla_{X} Y=\sum \pm\left\langle\nabla_{X} Y, e_{i}\right\rangle e_{i}+\frac{\left\langle\nabla_{X} Y, e_{n}\right\rangle}{\left\langle e_{n}, e_{n}\right\rangle} e_{n} .
$$

By transversality, the germ of $\left\langle e_{n}, e_{n}\right\rangle$ is a generator for the maximal ideal of the ring of germs at $p$. Hence, by an elementary division theorem, the last term extends smoothly if and only if $\left\langle\nabla_{X} Y, e_{n}\right\rangle=\square_{X} Y\left(e_{n}\right)$ vanishes over $S$. Since $e_{n}$ spans Rad over $S$ we are finished.

We now turn to the central result of this paper.

TheOREM 7. Given $(N,\langle\rangle) \pitchfork_{p} S(r, s)$ and $c:(-\varepsilon, \varepsilon) \rightarrow N$ a smooth curve transverse to the submanifold $S \subset N$ at $p=c(0)$. Let $\mathrm{II}^{\perp}(\dot{c})=\left\{v \in T_{p} N \mid \mathrm{II}_{p}(\dot{c}, v, R)=\right.$ $0\}$. Then for each $v$ in $\mathrm{II}^{\perp}(\dot{c})$ there exists a unique smooth vector field $P(t)$ along $c$ such that $D P / d t=0$ when $t \neq 0$ (classical covariant derivative along $c$ ) and $P(0)=v$.

We need the following lemma.

LEMMA 7.1. If $(N,\langle\rangle) \pitchfork_{p} S(r, s)$ of codimension one then the orthogonal complement of $\operatorname{Rad}_{p}$ relative to $\mathrm{II}_{p}(,, R)$ is $T_{p} S$. 
Proof. Choose a local radical adapated frame and $w_{p} \in T_{p} S$. Then $0=w_{p}\left\langle e_{n}, e_{n}\right\rangle$ $\equiv \mathrm{II}_{\mathrm{p}}(w, R, R)$. So $T_{p} S$ lies in the orthogonal complement of $\operatorname{Rad}_{p}$. Now Proposition 4 of [4] implies that it can be no larger.

Proof of Theorem 7. By the above lemma the subspaces $\mathrm{II}^{\perp}(\dot{c})$ and $\operatorname{Rad}_{p}$ split $T_{p} N$. Thus we may construct a radical adapted orthonormal frame $e_{1}(t) \cdots e_{n}(t)$ along the curve $c$ with $e_{1} \cdots e_{n-1}(0)$ spanning $\mathrm{II}^{\perp}(\dot{c})$. We may write $(D / d t) e_{i}=$ $a_{i j} e_{j}$ where all the $a_{i j}(t)$ are smooth except $a_{n n}=\square_{i} e_{n}\left(e_{n}\right) /\left\langle e_{n}, e_{n}\right\rangle$. But because $\mathrm{II}_{p}(\dot{c}, R, R) \neq 0, t \cdot a_{n n}(t)=b(t)$ extends smoothly to $t=0$ with $b(0)=\frac{1}{2}$. (This is the key observation and is a consequence of the accumulated transversality assumptions on the metric and curve.) We now view the o.d.e. for the parallel vector field $P$ as a vector field with "pole" $X$, defined on $c^{*} T N$ the pullback of the tangent bundle of $N$ to the curve. Via the frame $e_{i}$ we may identify $X$ with $\partial t-\sum^{n} x_{i} a_{i j}(t) \partial x_{j}$ on $\mathbf{R}^{n} \times(-\varepsilon, \varepsilon)$. We remove the "pole" in $X$ by considering $t \cdot X$ which is smooth and vanishes exactly on the locus $\left\{(x, t) \mid x_{n}=0\right.$ and $\left.t=0\right\}$. This locus identifies with $\mathrm{II}^{\perp}(\dot{c})$, so that $v \in \mathrm{II}^{\perp}(\dot{c})$ corresponds to a rest point of $t \cdot X$. The endomorphism associated to $t \cdot X$ at such a rest point may be identified with the $(n+1) \times(n+1)$ matrix,

$$
\left(\begin{array}{ccc}
0 & & 0 \\
& I & 0 \\
0 & 0 & -\frac{1}{2}
\end{array}\right)
$$

on the tangent space to $\mathbf{R}^{n} \times(-1,1)$. Thus $t \cdot X$ is a normally hyperbolic vector field (see the appendix). By the fundamental theorem of normally hyperbolic invariant manifolds we have for each rest point $v$ of $t X$, unique smooth 1-dimensional stable and unstable manifolds which intersect at the given rest point. The span of their tangent spaces at the intersection is transverse to the locus of rest points. The stable manifold suitably parametrized corresponds to the desired parallel vector field $P(t)$ along $c$.

The following two-dimensional illustrations provide some geometric intuition for the above. Recall that $X$, hence $t X$, must be tangent to the various sphere bundles over the curve, and that the spheres, $\left\{v \in T_{q} N \mid\langle v, v\rangle=c, c \in \mathbf{R}\right\}$ change topological structure as $q$ passes through $S$. See Figure 1. We visualize the spheres in $T_{c(t)} N$ as shown in Figure 2. So a sphere bundle in $c^{*} T N$ may be visualized as in Figure 3 and the trajectories of $t \cdot X$ which lie on a sphere bundle as in Figure 4 . The totality of extendable parallel vector fields in $c^{*} T N$ are as in Figure 5.

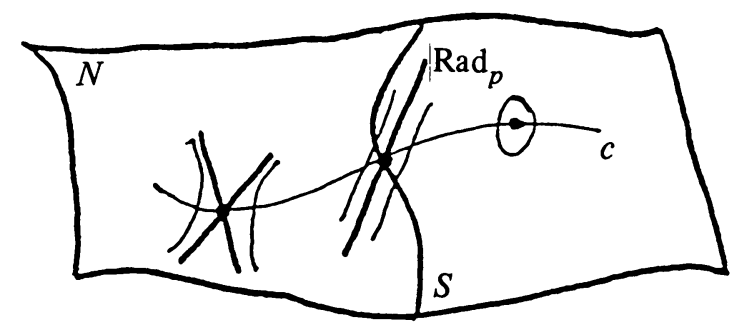

FIGURE 1 


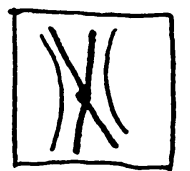

$t<0$

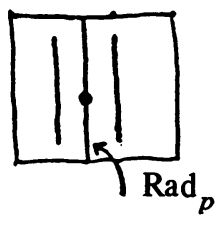

$t=0$

FIGURE 2
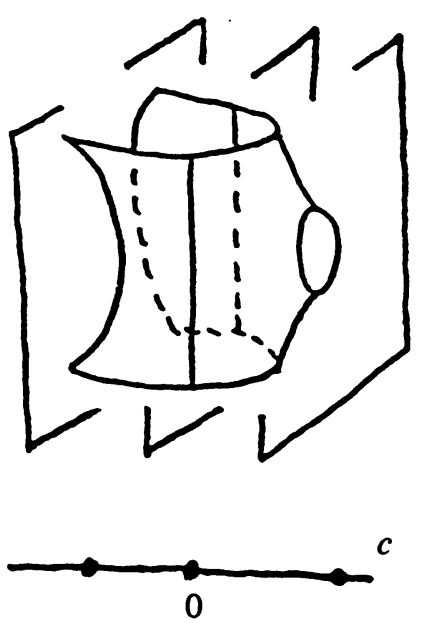

FIGURE 3

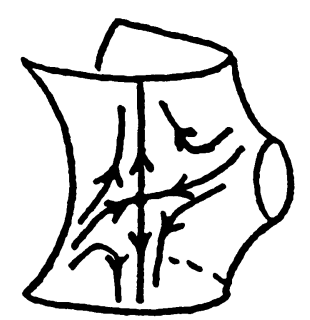

FIGURE 4

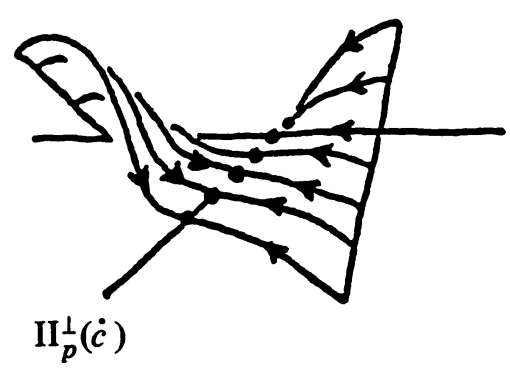

FIGURE 5

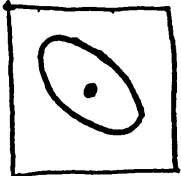

$t>0$ 
Applications. As an application of the above theorem consider the following analogy to the local de Rham decomposition encountered in nonsingular differential geometry. The proof is essentially a modification of the classical argument (compare Kobayashi, Nomizu, V. 1, p. 183). We say a distribution of r-planes is parallel along a curve if any parallel vector field along the curve initially tangent to the distribution remains tangent.

THEOREM 8. If $(N,\langle\rangle) \pitchfork_{p} S(r, s)$ admits a smooth $n-1$ distribution $W$ which is $C^{\infty}$ parallel along every curve transverse to the singular locus, then $N$ is locally isometric to the product of $S$ in its induced nonsingular $(r, s)$ metric with a one-dimensional transverse metric singularity.

Proof of Theorem 8. First we show that $W_{q}=T_{q} S$ for all $q$ in $S$ and that the radical must be transverse. First $W=\mathrm{II}^{\perp}(\dot{c})$ for all $\dot{c}$ transverse to $S$, whence for all $\dot{c}$ by continuity. Hence $\mathrm{II}_{q}(, R, R)$ vanishes on $W_{q}$. Thus by Lemma 6.1, $W_{p}=T_{q} S$. Now if the radical is not transverse it would then lie in $W_{q}$. But this contradicts the first part of Proposition 4.

Now we may apply the Gram-Schmidt algorithm to orthogonality and smoothly split the tangent bundle $T N$ as $W \oplus W^{\perp}$, where $W \oplus W^{\perp}=T S \oplus \operatorname{Rad}$ over $S$. Since this splitting is preserved under parallel transport along every curve transverse to the singular locus, it must be preserved along curves which lie entirely in one component of $N-S$. Now, it is classical that $W$ must then be involutive on $N-S$. Hence, by smoothness it is involutive on all of $N$. Now let $P_{i}, R, i=1, \ldots, n-1$, be a local coordinate frame at $p$ adapted to the splitting. Then $\operatorname{II}\left(P_{j}, R, R\right)=0$ and Proposition 6 implies that $\nabla_{R} P_{j}$ and $\nabla_{P_{j}} R$ extend smoothly to a neighborhood of $p$. By the invariance of the splitting we have $\nabla_{R} P_{j} \in W$ and $\nabla_{P_{j}} R \in R$. Thus, $0=$ $\left[P_{j}, R\right]=\nabla_{P_{j}} R+\nabla_{R} P_{j}$, hence $\nabla_{P_{j}} R=0=\nabla_{R} P_{j}$ on a neighborhood of $p$. It follows that $R\left\langle P_{i}, P_{j}\right\rangle=0$. Similarly, $P_{i}\langle R, R\rangle=0$ and we are finished.

We apply the above result to characterize flat metric singularities.

THEOREM 9. Given $(N,\langle\rangle) \pitchfork_{p} S(r, s)$ such that

(a) the curvature tensor vanishes on $N-S$,

(b) TS is the degenerate subbundle of the conformal structure II,

(c) the curvature tensor for $S$ in its induced metric connection vanishes.

Then $(N,\langle\rangle)$ is locally isometric to the product of a flat $(r, s)$ metric with a one-dimensional metric singularity.

Proof. Via (b) it is clear that the radical is transverse and, hence, that $S$ in its induced metric is nonsingular. Let $F_{i} \in V^{\infty}(S), i=1, \ldots, n-1$, be such that ${ }^{s} \nabla_{F_{i}} F_{j}=0$ for $i, j=1, \ldots, n-1$ and let $E_{i}, X \in V_{p}^{\infty}(N)$ be a local coordinate frame at $p$ such that $E_{i}=F_{i}$ over $S$. Now with (b) and Theorem 7 we may construct $P_{i} \in V^{\infty}(N)$ such that $P_{i}=E_{i}$ over $S$ and ${ }^{N} \nabla_{X} P_{i}=0, i=1, \ldots, n-1$, on a neighborhood of $p$. Therefore, on $N-S$ we have $0=K\left(X, E_{i}\right) P_{j}=\nabla_{X} \nabla_{E_{i}} P_{j}$ and hence by Proposition $6, \operatorname{II}\left(X, \nabla_{E_{i}}, P_{j}, R\right)=0$. By (b) we must have $\nabla_{E_{i}} P_{j} \in T S$ over $S$. The naturalness of dual connections with respect to submanifolds implies $\nabla_{E_{i}} P_{j}$ $={ }^{S} \nabla_{E_{i}} P_{j}={ }^{s} \nabla_{F_{i}} F_{j}=0$ over $S$. Thus, we must have $\nabla_{E_{i}} P_{j}=0$ on a neighborhood of 
$p$ in $N$ since the $E_{i}, X$ from a local frame $\square_{(} P_{i}()=0$ on a neighborhood of $p$. Now let $W$ be the span of the $P_{i}, i=1, \ldots, n-1$, and apply Theorem 8 .

EXAMPLES. Let $(V,\langle\rangle)$ be a 3-dimensional real vector space with bi-invariant $(2,1)$ metric. We will illustrate some of our results with hypersurfaces of $(V,\langle\rangle)$ which inherit transverse metric singularities. Let $x \in V$ be such that $\langle x, x\rangle=-1$. Choose $W \subset V$, a 2-dimensional subspace which contains $x$, and let $y \in W$ be of unit length and orthogonal to $x . R_{\theta}: V \rightarrow V$ will denote the 1-parameter group of orientation preserving isometries which fix $x$; and $T_{t, u}: V \rightarrow V$ will denote the 1-parameter group of translations in the direction $u \in V$. Now let $v:(-1,1) \rightarrow W$ be an imbedded curve.

(a) Cylinders. Consider $i(s, t)=T_{t, u}(v(s))$ where $u \notin W$. Then $i^{*}\langle\rangle$ is represented by $\langle\dot{v}, \dot{v}\rangle d s^{2}+\langle\dot{v}, u\rangle 2 d s d t+\langle u, u\rangle d t^{2}$. If $u \in W^{\perp}$, then

$$
\left((-1,1) \times R, i^{*}\langle\rangle\right) \pitchfork_{\left(s_{0}, t_{0}\right)} S(1,0)
$$

if and only if $\langle\dot{v}, \dot{v}\rangle_{s_{0}}=0$ and $d\langle\dot{v}, \dot{v}\rangle_{s_{0}} \neq 0$. Intuitively $v$ must be tangent to the null cone of $W$ in a transverse manner. In this case we have $S=\left\{(s, t) \mid s=s_{0}\right\}$ and $\operatorname{Rad}=\operatorname{span}(\partial s)$. Thus $\mathrm{II}_{s_{0}}(,, \partial s)$ is represented by $[(d / d s)\langle\dot{v}, \dot{v}\rangle] d s^{2}$. One readily sees that the hypothesis of Theorem $8(W \simeq \operatorname{span} \partial t)$ and Theorem 9 are satisfied. See Figure 6.

(b) Surfaces of revolution. Consider $i(s, \theta)=R_{\theta}(v(s))$ where $\langle v(s), y\rangle>0$. Then $i^{*}\langle\rangle$ is represented by $\langle\dot{v}, \dot{v}\rangle d s^{2}+\langle v, y\rangle^{2} d \theta^{2}$. It follows that $((-1,1) \times(-\pi, \pi)$, $\left.i^{*}\langle\rangle\right) \pitchfork_{\left(s_{0}, \theta_{0}\right)} S(1,0)$ if and only if $\langle\dot{v}, \dot{v}\rangle_{s_{0}}=0$ and $d\langle\dot{v}, \dot{v}\rangle_{s_{0}} \neq 0$. We have $S=$ $\left\{(s, \theta) \mid s=s_{0}\right\}$ and $\operatorname{Rad}=\operatorname{span}(\partial s)$. Here $\mathrm{II}_{s_{0}}(,, \partial s)$ is represented by $-(d / d s)\langle v, y\rangle d \theta^{2}+(d / d s)\langle\dot{v}, \dot{v}\rangle d s^{2}$. Transversality of the metric singularity implies that the intrinsic conformal structure has maximal rank. The type of the conformal structure is related to the convexity properties of the profile curve. The vector field $\partial \theta$ normalized to unit length is parallel along any meridian curve. See Figure 7.

(c) Helicoids. Consider $i(s, t)=T_{t, x} \circ R_{t}(v(s))$ where $\langle v(s), y\rangle>0$. Then $i^{*}\langle\rangle$ is represented by $\langle\dot{v}, \dot{v}\rangle d s^{2}+\langle x, v\rangle 2 d s d t+(\langle v, y\rangle-1) d t^{2}$. It follows that $((-1,1) \times(-1,1), \quad i *\langle\rangle) \pitchfork_{\left(s_{0}, t_{0}\right)} S(1,0)$ if $\langle x, v\rangle_{s_{0}}=0$ and $\langle v, y\rangle_{s_{0}}=1$ with $d\langle v, y\rangle_{s_{0}} \neq 0$. Notice that in this case the curve $v(s)$ is not metrically singular and
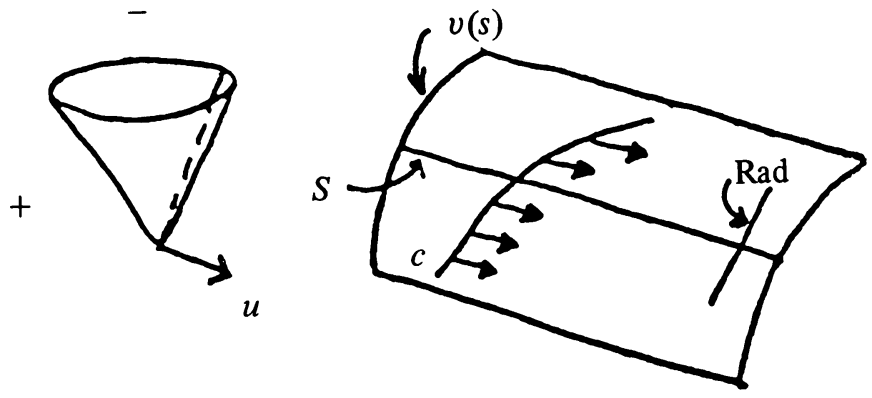

FIGURE 6 


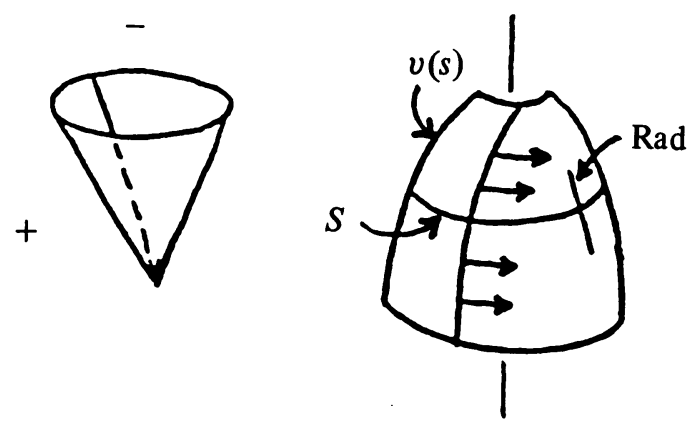

FIGURE 7
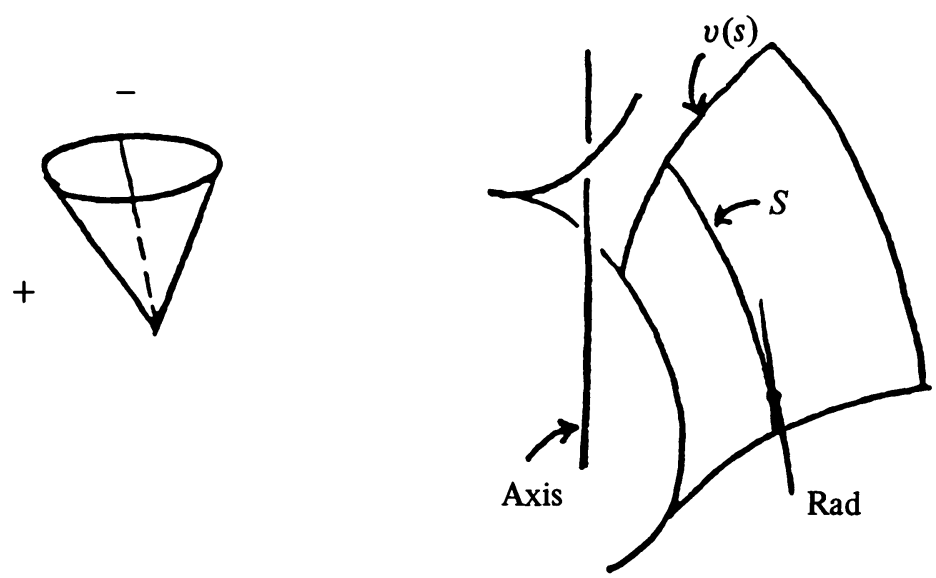

FIGURE 8

that the radical is nowhere transverse. Here the intrinsic conformal structure $\mathrm{II}_{s_{0}}(,, \partial t)$ is represented by $(d / d s)\langle x, \dot{v}\rangle d s^{2}+(d / d s)\langle y, \dot{v}\rangle d s d t$. In the case of the ruled helicoid (i.e. $\langle x, \dot{v}\rangle$ vanishes) the ruling lines are geodesics transverse to the singular locus. For such a ruling line $c$ we have $\mathrm{II}^{\perp}(\dot{c})=\dot{c}$ which together with $\partial t(\simeq \mathrm{Rad})$ split the tangent space. This illustrates Theorem 7 in the nonradical transverse case. See Figure 8.

Appendix. The fundamental theorem of normally hyperbolic invariant manifolds. Let $V$ be a smooth compact submanifold of a smooth manifold $M$. Let $f$ be a $C^{\infty}$ diffeomorphism of $M$ leaving $V$ invariant. We say $f$ is $\infty$-normally hyperbolic at $V$ iff the tangent bundle of $M$ restricted to $V$ splits into three smooth subbundles

$$
T M \mid V=N^{u} \oplus T V \oplus N^{s}
$$

invariant by $f_{*}$

$$
f_{*_{p}}=N_{p}^{u} f \oplus V_{p} f \oplus N_{p}^{s} f
$$


and there exists a Riemannian structure on $T M$ such that for all $p$ in $V$ and $k \geqslant 0$, $m\left(N^{u} f\right)>M\left(V_{p} f\right)^{k}$ and $M\left(N^{s} f\right)<m\left(V_{p} f\right)^{k}$. Here for a linear automorphism $A$, we write

$$
M(A)=\sup \{\|A(x)\|:\|x\|=1\}, \quad m(A)=\inf \{\|A(x)\|:\|x\|=1\} .
$$

THEOREM. If $f$ is $\infty$-normally hyperbolic at $V$ respecting $T M \mid V=N^{n} \oplus T V \oplus N^{s}$, then

(a) There exist locally $f$ invariant $C^{\infty}$ submanifolds $W^{u}$ and $W^{s}$ tangent at $V$ to $N^{u} \oplus T V$ and $T V \oplus N^{s}$ respectively.

(b) $W^{u}$ and $W^{s}$ are invariantly fibered by $C^{\infty}$ submanifolds $W_{p}^{u u}$ and $W_{p}^{s s}, p$ in $V$, tangent at $V$ to $N_{p}^{u}$ and $N_{p}^{s}$ respectively.

The theorem is applied to $C^{\infty}$ flows by considering a fixed time map. In the situation of Theorem 7, we are given $f_{*}$ of the form

$$
\left[\begin{array}{lllll}
1 & & & & 0 \\
& \ddots & & & \\
& & 1 & & \\
0 & & & a & \\
& & & & b
\end{array}\right]
$$

with $a>1>b>0$. The locus of rest points corresponds to the submanifold $\mathrm{II}^{\perp}(\dot{c})$.

\section{BIBLIOGRAPHY}

0. J. Beem, and P. Ehrlich, Global Lorentzian geometry, Dekker, New York, 1981.

1. M. Golubitsky and V. Guillemin, Stable mappings and their singularities, Graduate Texts in Math., Vol. 14, Springer-Verlag, 1973.

2. M. W. Hirsh, C. C. Pugh and M. Shub, Invariant manifolds, Lecture Notes in Math., vol. 583, Springer-Verlag, Berlin and New York, 1977.

3. S. Kobayasi and K. Nomizu, Foundations of differential geometry, Interscience, New York, 1969.

4. M. Kossowski, Fold singularities in pseudo Riemannian geodesic tubes, Proc. Amer. Math. Soc. 95 (1985), 463-469.

5.

6. B. O’Neill, Semi Riemannian geometry, Academic Press, 1983.

Department of Mathematics, Rice University, Houston, TeXas 77251 